\title{
Generalized cluster complexes via quiver representations
}

\author{
Bin Zhu
}

Received: 1 August 2006 / Accepted: 6 April 2007 /

Published online: 1 June 2007

(C) Springer Science+Business Media, LLC 2007

\begin{abstract}
We give a quiver representation theoretic interpretation of generalized cluster complexes defined by Fomin and Reading. Using $d$-cluster categories defined by Keller as triangulated orbit categories of (bounded) derived categories of representations of valued quivers, we define a $d$-compatibility degree $(-\|-)$ on any pair of "colored" almost positive real Schur roots which generalizes previous definitions on the noncolored case and call two such roots compatible, provided that their $d$ compatibility degree is zero. Associated to the root system $\Phi$ corresponding to the valued quiver, using this compatibility relation, we define a simplicial complex which has colored almost positive real Schur roots as vertices and $d$-compatible subsets as simplices. If the valued quiver is an alternating quiver of a Dynkin diagram, then this complex is the generalized cluster complex defined by Fomin and Reading.
\end{abstract}

Keywords Colored almost positive real Schur root - Generalized cluster complex · $d$-cluster category $\cdot d$-cluster tilting object $\cdot d$-compatibility degree

Mathematics Subject Classification (2000) 05A15 - 16G20 · 16G70 • 17B20

\section{Introduction}

Generalized cluster complexes associated to finite root systems are introduced by Fomin and Reading [12]. They have some nice properties, see [2] and references

Supported by the NSF of China (Grants 10471071) and by the Leverhulme Trust through the network 'Algebras, Representations and Applications'.

B. Zhu $(\bowtie)$

Department of Mathematical Sciences, Tsinghua University, 100084 Beijing, People's Republic of China

e-mail: bzhu@math.tsinghua.edu.cn 
therein. They are a generalization of cluster complexes (so-called generalized associahedra) associated to the same root systems introduced in [14, 15]. Cluster complexes describe the combinatorial structure of cluster algebras introduced by FominZelevinsky [13] in order to give an algebraic and combinatorial framework for the canonical basis, see [11] for a nice survey on this combinatorics and also cluster combinatorics of root systems. In [22], Marsh, Reineke and Zelevinsky use "decorated" quiver representations and tilting theory to give a quiver interpretation of cluster complexes. This connection between tilting theory and cluster combinatorics leads Buan, Marsh, Reineke, Reiten and Todorov [6] to introduce cluster categories for a categorical model for cluster algebras, see also [9] for type $A_{n}$. Cluster categories are the orbit categories $\mathcal{D} / \tau^{-1}[1]$ of derived categories of hereditary categories arising from the action of subgroup $\left\langle\tau^{-1}[1]\right\rangle$ of the automorphism group. They are triangulated categories [19] and now they have become a successful model for acyclic cluster algebras $[5,7,8]$, see also the surveys $[4,24]$ and references therein for recent developments and background of cluster tilting theory.

$d$-cluster categories $\mathcal{D} / \tau^{-1}[d]$, as a generalization of cluster categories, were introduced by Keller [19] and Thomas [25] for $d \in \mathbf{N}$. They are studied by Keller and Reiten [20], Palu [1, 23]; see also [3] for a geometric description of $d$-cluster categories of type $A_{n}$. $d$-cluster categories are triangulated categories with Calabi-Yau dimension $d+1$. When $d=1$, the cluster categories are recovered.

The aim of this paper is to give not only a quiver representation theoretic interpretation of all key ingredients in defining generalized cluster complexes using $d$-cluster categories, but also a generalization of generalized cluster complexes to infinite root systems (compare Remark 3.13 in [12], where the authors asked whether there was such an extension). For the simply-laced Dynkin case, Thomas [25] gives a realization of generalized cluster complexes by defining the $d$-cluster categories.

The paper is organized as follows: In the first two parts, we recall the well-known facts on $d$-cluster categories and (generalized) cluster complexes of finite root systems. In particular, we recall and generalize the BGP-reflection functors for cluster categories $[26,27]$ to $d$-cluster categories. In the third part, we prove some properties of $d$-cluster tilting objects, including that any basic $d$-cluster tilting object contains exactly $n$ indecomposable direct summands. In the final section, for any root system $\Phi$, using a $d$-cluster category $\mathcal{C}_{d}(\mathcal{H})$, we define a $d$-compatibility degree on any pair of colored almost positive real Schur roots. Using the $d$-compatibility degree, we define a generalized cluster complex associated to $\Phi$, which has colored almost positive real Schur roots as the vertices, and any subset forms a face if and only if any two elements of this subset are $d$-compatible. This simplicial complex is isomorphic to the cluster complex of $d$-cluster category $\mathcal{C}_{d}(\mathcal{H})$. If $\Phi$ is a finite root system, and if we take $\mathcal{H}_{0}$ to be the category of representations of an alternating quiver corresponding to $\Phi$, then our generalized cluster complex is the usual generalized cluster complex $\Delta^{d}(\Phi)$ defined by Fomin and Reading [12].

\section{Basics on $d$-cluster categories}

In this section, we collect some basic materials and fix the notation which we will use later on. 
A valued graph $(\Gamma, \mathbf{d})$ is a finite set of vertices $1, \ldots, n$, together with nonnegative integers $d_{i j}$ for all pairs $i, j \in \Gamma$ such that $d_{i i}=0$ and there exist positive integers $\left\{\varepsilon_{i}\right\}_{i \in \Gamma}$ satisfying

$$
d_{i j} \varepsilon_{j}=d_{j i} \varepsilon_{i} \quad \text { for all } i, j \in \Gamma \text {. }
$$

A pair $\{i, j\}$ of vertices is called an edge of $(\Gamma, \mathbf{d})$ if $d_{i j} \neq 0$. An orientation $\Omega$ of a valued graph $(\Gamma, \mathbf{d})$ is given by prescribing for each edge $\{i, j\}$ of $(\Gamma, \mathbf{d})$ an order (indicated by an arrow $i \rightarrow j$ ). For simplicity, we denote a valued graph by $\Gamma$ and a valued quiver by $(\Gamma, \Omega)$.

Let $(\Gamma, \Omega)$ be a valued quiver. We always assume that the valued quiver $(\Gamma, \Omega)$ contains no oriented cycles. Such orientation $\Omega$ is called admissible. Let $K$ be a field and $\mathbf{M}=\left(F_{i},{ }_{i} M_{j}\right)_{i, j \in \Gamma}$ a reduced $K$-species of $(\Gamma, \Omega)$; that is, for all $i, j \in \Gamma$, ${ }_{i} M_{j}$ is an $F_{i}-F_{j}$-bimodule, where $F_{i}$ and $F_{j}$ are division rings which are finitedimensional vector spaces over $K$ and $\operatorname{dim}\left({ }_{i} M_{j}\right)_{F_{j}}=d_{i j}$ and $\operatorname{dim}_{K} F_{i}=\varepsilon_{i}$. We denote by $\mathcal{H}$ the category of finite-dimensional representations of $(\Gamma, \Omega, \mathcal{M})$. It is a hereditary Abelian category [10]. Let $\Phi$ be the root system of the Kac-Moody Lie algebra corresponding to the graph $\Gamma$. We assume that $P_{1}, \ldots, P_{n}$ are nonisomorphic indecomposable projective representations in $\mathcal{H}, E_{1}, \ldots, E_{n}$ are simple representations with dimension vectors $\alpha_{1}, \ldots, \alpha_{n}$, and $\alpha_{1}, \ldots, \alpha_{n}$ are simple roots in $\Phi$. We use $D(-)$ to denote $\operatorname{Hom}_{K}(-, K)$, which is a duality of $\mathcal{H}$.

Denote by $\mathcal{D}=D^{b}(\mathcal{H})$ the bounded derived category of $\mathcal{H}$ with shift functor [1].

\section{$2.1 d$-cluster categories}

The derived category $\mathcal{D}$ has Auslander-Reiten triangles, and the Auslander-Reiten translate $\tau$ is an automorphism of $\mathcal{D}$. Fix a positive integer $d$ and denote $F_{d}=\tau^{-1}[d]$; it is an automorphism of $\mathcal{D}$. The $d$-cluster category of $H$ is defined in $[19,25]$ :

We denote by $\mathcal{D} / F_{d}$ the corresponding factor category. The objects are by definition the $F_{d}$-orbits of objects in $\mathcal{D}$, and the morphisms are given by

$$
\operatorname{Hom}_{\mathcal{D} / F_{d}}(\tilde{X}, \widetilde{Y})=\bigoplus_{i \in \mathbf{Z}} \operatorname{Hom}_{\mathcal{D}}\left(X, F_{d}^{i} Y\right) .
$$

Here $X$ and $Y$ are objects in $\mathcal{D}$, and $\tilde{X}$ and $\tilde{Y}$ are the corresponding objects in $\mathcal{D} / F_{d}$ (although we shall sometimes write such objects simply as $X$ and $Y$ ).

Definition 2.1 $([19,25])$ The orbit category $\mathcal{D} / F_{d}$ is called the $d$-cluster category of $\mathcal{H}$ (or of $(\Gamma, \Omega)$ ), which is denoted by $\mathcal{C}_{d}(\mathcal{H})$, sometimes denoted by $\mathcal{C}_{d}(\Omega)$.

By [19] the $d$-cluster category is a triangulated category with shift functor [1] which is induced by the shift functor in $\mathcal{D}$, the projection $\pi: \mathcal{D} \longrightarrow \mathcal{D} / F$ is a triangle functor. When $d=1$, this orbit category is called the cluster category of $\mathcal{H}$, denoted by $\mathcal{C}(\mathcal{H})$ (sometimes denoted by $\mathcal{C}(\Omega)$ ).

$\mathcal{H}$ is a full subcategory of $\mathcal{D}$ consisting of complexes concentrated in degree 0 , then passing to $\mathcal{C}_{d}(\mathcal{H})$ by the projection $\pi, \mathcal{H}$ is a (possibly, not full) subcategory of $\mathcal{C}_{d}(\mathcal{H})$. For any $i \in \mathbf{Z}$, we use $(\mathcal{H})[i]$ to denote the copy of $\mathcal{H}$ under the $i$ th shift $[i]$ as a subcategory of $\mathcal{C}_{d}(\mathcal{H})$. In this way, we have that (ind $\left.\mathcal{H}\right)[i]=\{M[i] \mid M \in$ ind $\mathcal{H}\}$. 
For any object $M$ in $\mathcal{C}_{d}(\mathcal{H})$, add $M$ denotes the full subcategory of $\mathcal{C}_{d}(\mathcal{H})$ consisting of direct summands of direct sums of copies of $M$.

For $X, Y \in \mathcal{C}_{d}(\mathcal{H})$, we will use $\operatorname{Hom}(X, Y)$ to denote the Hom-space $\operatorname{Hom}_{\mathcal{C}_{d}(\mathcal{H})}(X, Y)$ in the $d$-cluster category $\mathcal{C}_{d}(\mathcal{H})$ throughout the paper. Define $\operatorname{Ext}^{i}(X, Y)$ to be $\operatorname{Hom}(X, Y[i])$.

We summarize some known facts about $d$-cluster categories $[6,19]$.

Proposition 2.2 (1) $\mathcal{C}_{d}(\mathcal{H})$ has Auslander-Reiten triangles and Serre functor $\Sigma=$ $\tau[1]$, where $\tau$ is the AR-translate in $\mathcal{C}_{d}(\mathcal{H})$, which is induced from AR-translate in $\mathcal{D}$.

(2) $\mathcal{C}_{d}(\mathcal{H})$ is a Calabi-Yau category of $C Y$-dimension $d+1$.

(3) $\mathcal{C}_{d}(\mathcal{H})$ is a Krull-Remark-Schmidt category.

(4) ind $\mathcal{C}_{d}(\mathcal{H})=\bigcup_{i=0}^{i=d-1}($ ind $\mathcal{H})[i] \cup\left\{P_{j}[d] \mid 1 \leq j \leq n\right\}$.

Proof (1) This is Proposition 1.3 of [6] and Corollary 1 in Sect. 8.4 of [19].

(2) It is proved in Corollary 1 in Sect. 8.4 of [19].

(3) This is proved in Proposition 1.2 of [6].

(4) The proof for $d=1$ is given in Proposition 1.6 of [6], which can be modified for the general $d$.

From Proposition 2.2 we define the degree for every indecomposable object in $\mathcal{C}_{d}(\mathcal{H})$ as follows:

Definition 2.3 For any indecomposable object $X \in \mathcal{C}_{d}(\mathcal{H})$, we call the nonnegative integer $\min \left\{k \in \mathbf{Z}_{\geq 0} \mid X \cong M[k]\right.$ in $\mathcal{C}_{d}(\mathcal{H})$ for some $M \in$ ind $\left.\mathcal{H}\right\}$ the degree of $X$, denoted by $\operatorname{deg} X$.

By Definition 2.3 any indecomposable object $X$ of degree $k$ is isomorphic to $M[k]$ in $\mathcal{C}_{d}(\mathcal{H})$, where $M$ is an indecomposable representation in $\mathcal{H} ; 0 \leq \operatorname{deg} X \leq d, X$ has degree $d$ if and only if $X \cong P[d]$ in $\mathcal{C}_{d}(\mathcal{H})$ for some indecomposable projective object $P \in \mathcal{H}$; and $X$ has degree 0 if and only if $X \cong M[0]$ in $\mathcal{C}_{d}(\mathcal{H})$ for some indecomposable object $M \in \mathcal{H}$. Here $M[0]$ means regarding the object $M$ of $\mathcal{H}$ as a complex concentrated in degree 0 .

\subsection{BGP-reflection functors}

If $T$ is a tilting object in $\mathcal{H}$, then the endomorphism algebra $A=\operatorname{End}_{\mathcal{H}}(T)$ is called a tilted algebra. The tilting functor $\operatorname{Hom}_{\mathcal{H}}(T,-)$ induces the equivalence $\operatorname{RHom}(T,-): D^{b}(\mathcal{H}) \rightarrow D^{b}(A)$, where $\operatorname{RHom}(T,-)$ is the derived functor of $\operatorname{Hom}_{\mathcal{H}}(T,-)$.

Any standard triangle functor $G: D^{b}(\mathcal{H}) \rightarrow D^{b}\left(\mathcal{H}^{\prime}\right)$ induces a well-defined functor $\tilde{G}: \mathcal{C}_{d}(\mathcal{H}) \longrightarrow \mathcal{C}_{d}\left(\mathcal{H}^{\prime}\right)$ with the following commutative diagram [19, 26]:

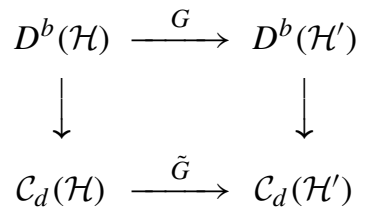

The following result is proved in [26, 27]. 
Proposition 2.4 If $G: D^{b}(\mathcal{H}) \rightarrow D^{b}\left(\mathcal{H}^{\prime}\right)$ is a triangle equivalence, then $\tilde{G}$ is also an equivalence of triangulated categories.

Let $k$ be a vertex in the valued quiver $(\Gamma, \Omega)$; the reflection of $(\Gamma, \Omega)$ at $k$ is the valued quiver $\left(\Gamma, s_{k} \Omega\right)$, where $s_{k} \Omega$ is the orientation of $\Gamma$ obtained from $\Omega$ by reversing all arrows starting or ending at $k$. The corresponding category of representations of $\left(\Gamma, s_{k} \Omega, \mathcal{M}\right)$ is denoted simply by $s_{k} \mathcal{H}$. If $k$ is a sink in the valued quiver $(\Gamma, \Omega)$, then $k$ is a source of $\left(\Gamma, s_{k} \Omega\right)$, and the reflection of $\left(\Gamma, s_{k} \Omega\right)$ at $k$ is $(\Gamma, \Omega)$. Let $k$ be a sink in $(\Gamma, \Omega)$. Then $P_{k}$ is a simple projective representation, and $T=\oplus_{j \neq k} P_{j} \oplus \tau^{-1} P_{k}$ is a tilting representation in $\mathcal{H}$ [24]. The tilting functor $S_{k}^{+}=\operatorname{Hom}_{\mathcal{H}}(T,-)$ is a so-called BGP-reflection functor, and its derived functor $\operatorname{RHom}(T,-)$ is a triangle equivalence from $D^{b}(\mathcal{H})$ to $D^{b}\left(s_{k} \mathcal{H}\right)$, which is also denoted by $S_{k}^{+}$. Similarly, one has BGP-reflection functors $S_{k}^{-}$for sources $k$.

Definition 2.5 The induced functors $\widetilde{S_{k}^{+}}: \mathcal{C}_{d}(\mathcal{H}) \longrightarrow \mathcal{C}_{d}\left(s_{k} \mathcal{H}\right)$ for sinks $k$ and $\widetilde{S_{k}^{-}}$: $\mathcal{C}_{d}(\mathcal{H}) \longrightarrow \mathcal{C}_{d}\left(s_{k} \mathcal{H}\right)$ for sources $k$ are called BGP-reflection functors of $d$-cluster categories.

Remark 2.6 When $d=1$, BGP-reflection functors are discussed in [26].

We remind the reader that $\mathcal{H}$ (or $\mathcal{H}^{\prime}$ ) is the category of representations of the valued quiver $(\Gamma, \Omega)\left(\left(\Gamma, s_{k} \Omega\right)\right.$, respectively); the $P_{i}$ (respectively, the $\left.P_{i}^{\prime}\right)$ are the indecomposable projective representations in $\mathcal{H}$ (respectively, $\mathcal{H}^{\prime}$ ), and the $E_{i}$ (respectively, the $E_{i}^{\prime}$ ) are the corresponding simple representations which are the tops of the $P_{i}$ (respectively, the $P_{i}^{\prime}$ ) for $i=1, \ldots, n$.

We recall from Proposition 2.2 and Definition 2.3 that any indecomposable object $Y$ in $\mathcal{C}_{d}(\mathcal{H})$ is isomorphic to $X[i]$, where $X \in$ ind $\mathcal{H}$, and $i$ is the degree of $Y$. Keeping this notation, we have the following proposition which gives the images of indecomposable objects in $\mathcal{C}_{d}(\mathcal{H})$ under the BGP-reflection functor $\widetilde{S_{k}^{+}}$.

Proposition 2.7 Let $k$ be a sink of the valued quiver $(\Gamma, \Omega)$ and $Y$ an indecomposable object in $\mathcal{C}_{d}(\mathcal{H})$ with degree $i$. Then $Y \cong X[i]$ for an indecomposable representation $X$ in $\mathcal{H}$, and

$$
\widetilde{S_{k}^{+}}(X[i])= \begin{cases}P_{k}^{\prime}[d] & \text { if } \left.X \cong P_{k} \cong E_{k}\right) \text { and } i=0, \\ E_{k}^{\prime}[i-1] & \text { if } X \cong P_{k}\left(\cong E_{k}\right) \text { and } 0<i \leq d, \\ P_{j}^{\prime}[d] & \text { if } X \cong P_{j} \nsucceq P_{k} \text { and } i=d, \\ S_{k}^{+}(X)[i] & \text { otherwise. }\end{cases}
$$

Proof The statement in the proposition was proved in $[26,27]$ when $d=1$. The proof for the case $d>1$ is the same as there. We give a sketch of the proof for the convenience of readers. The BGP-reflection functor $S_{k}^{+}: \mathcal{H} \longrightarrow s_{k} \mathcal{H}$ induces a triangle equivalence $D^{b}(\mathcal{H}) \longrightarrow D^{b}\left(s_{k} \mathcal{H}\right)$, denoted also by $S_{k}^{+}$. It induces an equivalence ind $D^{b}(\mathcal{H}) \longrightarrow$ ind $D^{b}\left(s_{k} \mathcal{H}\right)$. For any indecomposable object $X[i] \in$ ind $D^{b}(\mathcal{H})$, it is not hard to show that $S_{k}^{+}(X[i])=S_{k}^{+}(X)[i]$ for $X ¥ P_{k}$ (note that $P_{k}=E_{k}$, since 
$k$ is a sink in $(\Gamma, \Omega)$ ), and $S_{k}^{+}\left(P_{k}[i]\right)=E_{k}^{\prime}[i-1]$ for $i \in \mathbf{Z}$ (cf. [26] or [27]). Since $E_{k}^{\prime}$ is an injective representation in $s_{k} \mathcal{H}$, we have $\tau P_{k}^{\prime}[i]=E_{k}^{\prime}[i-1]$ in $D^{b}\left(s_{k} \mathcal{H}\right)$. Now passing to the $d$-cluster category $\mathcal{C}_{d}(\mathcal{H})$ (which is an orbit category of the derived category $D^{b}(\mathcal{H})$ ), we get the images of indecomposable objects of $\mathcal{C}_{d}(\mathcal{H})$ under $\widetilde{S_{k}^{+}}$ as stated in the proposition.

\section{Cluster combinatorics of root systems}

For a valued graph $\Gamma$, we denote by $\Phi=\Phi^{+} \cup \Phi^{-}$the set of roots of the corresponding Kac-Moody Lie algebra.

Definition 3.1 (1) The set of almost positive roots is

$$
\Phi_{\geq-1}=\Phi^{+} \cup\left\{-\alpha_{i} \mid i=1, \ldots n\right\} .
$$

(2) Denote by $\Phi_{\geq-1}^{\text {re }}$ the subset of $\Phi_{\geq-1}$ consisting of the positive real roots together with the negatives of the simple roots.

When $\Phi$ is of finite type, $\Phi_{\geq-1}=\Phi_{\geq-1}^{\text {re }}$.

Definition 3.2 Let $s_{i}$ be the Coxeter generator of the Weyl group of $\Phi$ corresponding to $i \in \Gamma_{0}$. We call the following map the "truncated simple reflection" $\sigma_{i}$ of $\Phi_{\geq-1}$ [14]:

$$
\sigma_{i}(\alpha)= \begin{cases}\alpha, & \alpha=-\alpha_{j}, j \neq i \\ s_{i}(\alpha), & \text { otherwise }\end{cases}
$$

It is easy to see that $\sigma_{i}$ is an automorphism of $\Phi_{\geq-1}^{\mathrm{re}}$.

\subsection{Cluster complexes of finite root systems}

In this first paragraph, we do not assume that $\Gamma$ is a Dynkin diagram (i.e., of finite type). Let $i_{1}, \ldots, i_{n}$ be an admissible ordering of $\Gamma$ with respect to $\Omega$, i.e., $i_{t}$ is a sink with respect to $s_{i_{t-1}} \cdots s_{i_{2}} s_{i_{1}} \Omega$ for any $1 \leq t \leq n$. Denote $R_{\Omega}=\sigma_{i_{n}} \cdots \sigma_{i_{1}}$. This is an automorphism of $\Phi_{\geq-1}$ and does not depend on the choice of admissible ordering of $\Gamma$ with respect to $\Omega$. It is the automorphism induced by the Auslander-Reiten translation $\tau$ in $\mathcal{C}(\mathcal{H})$ (cf. [26, 27]).

In the rest of this subsection, we always assume that $\Gamma$ is a valued Dynkin graph, which is not necessarily connected. Fomin and Zelevinsky [15] associate a nonnegative integer $(\alpha \| \beta)$, known as the compatibility degree, to each pair $\alpha, \beta$ of almost positive roots.

This is defined in the following way: Let $\Omega_{0}$ denote one of the alternating orientations of $\Gamma$, and $\Gamma^{+}$(respectively, $\Gamma^{-}$) the set of sinks (respectively, sources) of $\left(\Gamma, \Omega_{0}\right)$. Define

$$
\tau_{ \pm}=\prod_{i \in \Gamma^{ \pm}} \sigma_{i}
$$


Then $R_{\Omega_{0}}=\tau_{-} \tau_{+}$, which is simply denoted by $R$.

Denote by $n_{i}(\beta)$ the coefficient of $\alpha_{i}$ in the expansion of $\beta$ in terms of the simple roots $\alpha_{1}, \ldots, \alpha_{n}$. Then (\|) is uniquely defined by the following two properties:

$$
\begin{array}{ll}
(*) & \left(-\alpha_{i} \| \beta\right)=\max \left(\left[\beta: \alpha_{i}\right], 0\right), \\
(* *) & \left(\tau_{ \pm} \alpha \| \tau_{ \pm} \beta\right)=(\alpha \| \beta),
\end{array}
$$

for any $\alpha, \beta \in \Phi_{\geq-1}$ and any $i \in \Gamma$.

Two almost positive roots $\alpha, \beta$ are called compatible if $(\alpha \| \beta)=0$.

The cluster complex $\Delta(\Phi)$ associated to the finite root system $\Phi$ is defined in [14].

Definition 3.3 The cluster complex $\Delta(\Phi)$ is a simplicial complex on the ground set $\Phi_{\geq-1}$. Its faces are mutually compatible subsets of $\Phi_{\geq-1}$. The facets of $\Delta(\Phi)$ are called the (root-)clusters associated to $\Phi$.

\subsection{Generalized cluster complexes of finite root systems}

At the beginning of this subsection, we assume that $\Gamma$ is an arbitrary valued graph, which is not necessarily connected, except where we express specifically. As before, $\Phi$ denotes the set of roots of the corresponding Lie algebra, and $\Phi_{\geq-1}$ denotes the set of almost positive roots. Fix a positive integer $d$; for any $\alpha \in \Phi^{+}$, following [12], we call $\alpha^{1}, \ldots, \alpha^{d}$ the $d$ "colored" copies of $\alpha$.

Definition 3.4 ([12]) The set of colored almost positive roots is

$$
\Phi_{\geq-1}^{d}=\left\{\alpha^{i}: \alpha \in \Phi_{>0}, i \in\{1, \ldots, d\}\right\} \cup\left\{\left(-\alpha_{i}\right)^{1}: 1 \leq i \leq n\right\} .
$$

When $\Gamma$ is a Dynkin graph, the root system $\Phi$ of the corresponding Lie algebra is finite. In this case, the generalized cluster complex $\Delta^{d}(\Phi)$ is defined on the ground set $\Phi_{\geq-1}^{d}$ and using the binary compatibility relation on $\Phi_{>-1}^{d}$. This binary compatibility relation is a natural generalization of binary compatibility relation on $\Phi_{\geq-1}$, which we now recall from [12].

For a root $\beta \in \Phi_{\geq-1}$, let $t(\beta)$ denote the smallest $t$ such that $R^{t}(\beta)$ is a negative root.

Definition 3.5 ([12]) Two colored roots $\alpha^{k}, \beta^{l} \in \Phi_{\geq-1}^{d}$ are called compatible if and only if one of the following conditions is satisfied:

(1) $k>l . t(\alpha) \leq t(\beta)$, and the roots $R(\alpha)$ and $\beta$ are compatible (in the original "non-colored" sense).

(2) $k<l$. $t(\alpha) \geq t(\beta)$, and the roots $\alpha$ and $R(\beta)$ are compatible.

(3) $k>l . t(\alpha)>t(\beta)$, and the roots $\alpha$ and $\beta$ are compatible.

(4) $k<l$. $t(\alpha)<t(\beta)$, and the roots $\alpha$ and $\beta$ are compatible.

(5) $k=l$. And the roots $\alpha$ and $\beta$ are compatible.

Now we are ready to recall the definition of generalized cluster complex $\Delta^{d}(\Phi)$ for a finite root system $\Phi$. 
Definition $3.6([12]) \Delta^{d}(\Phi)$ has $\Phi_{\geq-1}^{d}$ as the set of vertices, its simplices are mutually compatible subsets of $\Phi_{\geq-1}^{d}$. The subcomplex of $\Delta^{d}(\Phi)$ which has $\Phi_{>0}^{d}$ as the set of vertices is denoted by $\Delta_{+}^{d}(\Phi)$

Now we generalize the definition of $R_{d}$ [12] for a finite root system to an arbitrary root system.

Definition 3.7 Let $(\Gamma, \Omega)$ be a valued quiver. For $\alpha^{k} \in \Phi_{\geq-1}^{d}$, we set

$$
R_{d, \Omega}\left(\alpha^{k}\right)= \begin{cases}\alpha^{k+1} & \text { if } \alpha \in \Phi_{>0} \text { and } k<d \\ \left(R_{\Omega}(\alpha)\right)^{1} & \text { otherwise }\end{cases}
$$

Remark 3.8 If $\left(\Gamma, \Omega_{0}\right)$ is a valued Dynkin graph with an alternating orientation, then the automorphism $R$ of $\Phi_{\geq-1}$ defined by Fomin and Zelevinsky [14] is $R_{\Omega_{0}}$; hence, $R_{d, \Omega_{0}}$ is the usual one $\left(R_{d}\right)$ defined by Fomin and Reading [12].

Theorem 3.9 ([12]) Let $\Phi$ be a finite root system. The compatibility relation on $\Phi_{\geq-1}^{d}$ has the following properties:

(1) $\alpha^{k}$ is compatible with $\beta^{l}$ if and only if $R_{d}\left(\alpha^{k}\right)$ is compatible with $R_{d}\left(\beta^{l}\right)$.

(2) $\left(-\alpha_{i}\right)^{1}$ is compatible with $\beta^{l}$ if and only if $n_{i}(\beta)=0$.

Moreover, conditions 1-2 uniquely determine this relation.

Now we generalize the "truncated simple reflections" of $\Phi_{\geq-1}$ to the colored almost positive roots. Let $\Phi$ be an arbitrary root system (not necessarily of finite type).

Definition 3.10 Let $s_{k}$ be the Coxeter generator of the Weyl group of $\Phi$ corresponding to $k \in \Gamma_{0}$. We define the following map $\sigma_{k, d}$ of $\Phi_{\geq-1}^{d}$ :

$$
\sigma_{k, d}\left(\alpha^{i}\right)= \begin{cases}\alpha_{k}^{d} & \text { if } i=1 \text { and } \alpha=-\alpha_{k} \\ \alpha_{k}^{i-1} & \text { if } 1<i \leq d \text { and } \alpha=\alpha_{k} \\ \left(-\alpha_{j}\right)^{1} & \text { if } i=1 \text { and } \alpha=-\alpha_{j}, j \neq k \\ \left(s_{k}(\alpha)\right)^{i} & \text { otherwise. }\end{cases}
$$

$\sigma_{k, d}$ is a bijection of $\Phi_{\geq-1}^{d}$. We call it a $d$-truncated simple reflection of $\Phi_{\geq-1}^{d}$.

\section{$4 d$-cluster tilting in $d$-cluster categories}

Let $\mathcal{C}_{d}(\mathcal{H})$ be a $d$-cluster category of type $\mathcal{H}$, where $\mathcal{H}$ is the category of representations of the valued quiver $(\Gamma, \Omega)$. It is a Calabi-Yau triangulated category with CY-dimension $d+1$.

Definition 4.1 (1) An object $X$ in $\mathcal{C}_{d}(\mathcal{H})$ is called exceptional if $\operatorname{Ext}^{i}(X, X)=0$ for any $1 \leq i \leq d$. 
(2) An object $X$ is called a $d$-cluster tilting object if it satisfies the property: $Y \in \operatorname{add}(X)$ if and only if $\operatorname{Ext}^{i}(X, Y)=0$ for $1 \leq i \leq d$.

(3) An object $X$ is called almost complete tilting if there is an indecomposable object $Y$ such that $X \oplus Y$ is a $d$-cluster tilting object. Such an indecomposable object $Y$ is called a complement of $X$.

Proposition 4.2 (1) For an object $X$ in $\mathcal{H}, X$ is exceptional in $\mathcal{H}$ i.e., $\operatorname{Ext}_{\mathcal{H}}^{1}(X, X)=$ 0 if and only if $X[0]$ is exceptional in $\mathcal{C}_{d}(\mathcal{H})$.

(2) Any indecomposable exceptional object $X$ in $\mathcal{C}_{d}(\mathcal{H})$ is of the form $M[i]$ with $M$ being an exceptional representation in $\mathcal{H}$ and $0 \leq i \leq d-1$ or of the form $P_{j}[d]$ for some $1 \leq j \leq n$. In particular, if $\Gamma$ is a Dynkin graph, then any indecomposable object in $\mathcal{C}_{d}(\mathcal{H})$ is exceptional.

(3) Suppose that $d>1$. Then $\operatorname{End}_{\mathcal{C}_{d}(\mathcal{H})} X$ is a division algebra for any indecomposable exceptional object $X$.

(4) Suppose that $d>1$. Let $P$ be a projective representation in $\mathcal{H}$ and $X$ a representation in $\mathcal{H}$. Then, for any $-d \leq i \leq d, \operatorname{Ext}^{1}(P, X[i])=0$ except possibly for $i \in\{-1, d-1, d\}$.

Proof (1) Let $X \in \mathcal{H}$ be exceptional. We will prove that $\operatorname{Ext}^{i}(X, X)=0$ for any $i \in$ $\{1, \ldots, d\}$. By definition we have that $\operatorname{Ext}^{i}(X, X)=\bigoplus_{k \in \mathbf{Z}} \operatorname{Ext}_{\mathcal{D}}^{i}\left(X, \tau^{-k} X[k d]\right)=$ $\operatorname{Ext}_{\mathcal{D}}^{i}(X, X) \oplus \operatorname{Ext}_{\mathcal{D}}^{i}(X, \tau X[-d])$. In this sum, the first summand $\operatorname{Ext}_{\mathcal{D}}^{i}(X, X)=$ $0, \forall i \geq 1$, while the second summand $\operatorname{Ext}_{\mathcal{D}}^{i}(X, \tau X[-d]) \cong \operatorname{Hom}_{\mathcal{D}}(X, \tau X[i-d])$, which is zero when $i<d$ and is isomorphic to $\operatorname{Ext}_{\mathcal{D}}^{1}(X, X)=0$ when $i=d$. This proves that $X$ is exceptional in $\mathcal{C}_{d}(\mathcal{H})$. The proof for the converse directly follows from the definition.

(2) The statements follow from Proposition 2.2(4) and Definition 4.1, also using Part 1 and the fact that the shift is an autoequivalence.

(3) Let $X$ be an indecomposable exceptional representation in $\mathcal{H}$, and suppose that $d>1$. From the definition of the orbit category It follows that $\operatorname{End}_{\mathcal{C}_{d}(H)} X \cong$ $\bigoplus_{m \in \mathbf{Z}} \operatorname{Hom}_{\mathcal{D}}\left(X, \tau^{-m} X[d m]\right) \cong \operatorname{End}_{\mathcal{H}} X$. The last isomorphism holds due to the facts: $\operatorname{Hom}_{\mathcal{D}}\left(X, \tau^{m} X[-m d]\right) \cong \operatorname{Hom}_{\mathcal{D}}\left(X[m d], \tau^{m} X\right) \cong \operatorname{Ext}_{\mathcal{D}}^{1}\left(\tau^{m-1} X, X[m d]\right)=$ 0 for any positive integer $m$ and $\operatorname{Hom}_{\mathcal{D}}\left(X, \tau^{-m} X[m d]\right) \cong \operatorname{Hom}_{\mathcal{D}}\left(\tau^{m} X, X[m d]\right)$, which is also zero, since $m d>1$ (we use the assumption $d>1$ here) for any positive integer $m$. Then $\operatorname{End}_{\mathcal{C}_{d}(H)} X$ is a division algebra, since $\operatorname{End}_{\mathcal{H}} X$ is a division algebra. Since any indecomposable exceptional object $M$ in $\mathcal{C}_{d}(H)$ is some shift $X[i]$ of an indecomposable exceptional representation $X$ in $\mathcal{H}, \operatorname{End}_{\mathcal{C}_{d}(H)} M=\operatorname{End}_{\mathcal{C}_{d}(H)} X[i] \cong$ End $_{\mathcal{C}_{d}(H)} X$ is a division algebra.

(4) Suppose that $d>1$. Let $P$ be a projective representation in $\mathcal{H}$ and $X$ a representation in $\mathcal{H}$. Then, for any $-d \leq i \leq d, \operatorname{Ext}^{1}(P, X[i])=$ $\bigoplus_{k \in \mathbf{Z}} \operatorname{Ext}_{\mathcal{D}}^{1}\left(P, \tau^{-k} X[d k+i]\right) \cong \operatorname{Ext}_{\mathcal{D}}^{1}(P, \tau X[-d+i]) \oplus \operatorname{Ext}_{\mathcal{D}}^{1}(P, X[i])$. Now if $i \neq-1, d-1, d$, then $\operatorname{Ext}_{\mathcal{D}}^{1}(P, \tau X[-d+i])=0=\operatorname{Ext}_{\mathcal{D}}^{1}(P, X[i])$. Then, for any $-d \leq i \leq d, \operatorname{Ext}^{1}(P, X[i])=0$ except for $i=-1, d-1$, and $d$.

Remark 4.3 Any basic (i.e., multiplicity-free) exceptional object contains at most $(d+1) n$ nonisomorphic indecomposable direct summands. 
Proof Let $X$ be a basic exceptional object in $\mathcal{C}_{d}(\mathcal{H})$. Then any indecomposable direct summand of $X$ is exceptional; hence, by Proposition 4.2(2), we write $M$ as $M=\bigoplus_{k=0}^{k=d} \bigoplus_{i \in I_{k}} M_{i, k}[k]$ with $M_{i, k}$ being an indecomposable exceptional representation. Therefore, $\bigoplus_{i \in I_{k}} M_{i, k}$ is an exceptional object in hereditary category $\mathcal{H}$; hence, the number of direct summands is at most $n$, i.e., $\left|I_{k}\right| \leq n$. Then the number of indecomposable direct summands of $M$ is at most $(d+1) n$.

For any pair of objects $T, X$ in $\mathcal{C}_{d}(\mathcal{H})$, due to the Calabi-Yau property of $\mathcal{C}_{d}(\mathcal{H})$, we have that $\operatorname{Ext}^{i}(X, T)=0$ for $1 \leq i \leq d$ if and only if $\operatorname{Ext}^{i}(T, X)=0$ for $1 \leq i \leq d$. Hence, by Remark 4.3 and Definition 4.1, $T$ is a $d$-cluster tilting object in $\mathcal{C}_{d}(\mathcal{H})$ if and only if add $T$ is a maximal $d$-orthogonal subcategory of $\mathcal{C}_{d}(\mathcal{H})$ in the sense of [17]: i.e., add $T$ is contravariantly finite and covariantly finite in $\mathcal{C}_{d}(\mathcal{H})$ and satisfies the following property: $X \in \operatorname{add} T$ if and only if $\operatorname{Ext}^{i}(X, T)=0$ for $1 \leq i \leq d$ if and only if $\operatorname{Ext}^{i}(T, X)=0$ for $1 \leq i \leq d$. In the following, we will prove that any basic $d$ cluster tilting object contains exactly $n$ indecomposable direct summands. First of all, we recall some results from [17] which hold in any $(d+1)$-Calabi-Yau triangulated category.

Theorem 4.4 (Iyama) Let $X$ be an almost complete tilting object in $\mathcal{C}_{d}(\mathcal{H})$ and $X_{0}$ a complement of $X$. Then there are $d+1$ triangles:

$$
\text { (*) } \quad X_{i+1} \stackrel{g_{i}}{\longrightarrow} B_{i} \stackrel{f_{i}}{\longrightarrow} X_{i} \stackrel{\sigma_{i}}{\longrightarrow} X_{i+1}[1]
$$

where $f_{i}$ is the minimal right add $X$-approximation of $X_{i}$ and $g_{i}$ minimal left add $X$-approximation of $X_{i+1}$, all $X_{i}$ are indecomposable and complements of $X$, $i=0, \ldots, d$.

For the convenience of readers, we sketch the proof; for details, see [18].

Proof We suppose that $d>1$; the same statement for $d=1$ was proved in [6]. For the complement $X_{0}$ of $X$, we consider the minimal right add $X$-approximation $f_{0}$ : $B_{0} \rightarrow X_{0}$ of $X_{0}$, extend $f_{0}$ to the triangle $X_{1} \stackrel{g_{0}}{\rightarrow} B_{0} \stackrel{f_{0}}{\rightarrow} X_{0} \stackrel{\sigma_{0}}{\rightarrow} X_{1}[1]$. It is easy to see that $X_{1}$ is indecomposable, $g_{0}$ is the minimal left add $X$-approximation of $X_{1}$, and $X \oplus X_{1}$ is an exceptional object in $\mathcal{C}_{d}(\mathcal{H})$ (cf. [6]). From Theorem 5.1 in [18] it follows that $X \oplus X_{1}$ is a $d$-cluster tilting object. Continuing this step, one can get complements $X_{1}, \ldots, X_{d+1}$ with triangles $X_{i+1} \stackrel{g_{i}}{\rightarrow} B_{i} \stackrel{f_{i}}{\rightarrow} X_{i} \stackrel{\sigma_{i}}{\rightarrow} X_{i}[1]$ for $0 \leq i \leq d$, where $f_{i}\left(g_{i}\right)$ is the minimal right (left, resp.) add $X$-approximation of $X_{i}\left(X_{i+1}\right.$, resp.), and $X \oplus X_{i}$ is a $d$-cluster tilting object.

Corollary 4.5 With the notation of Theorem 4.4 , we have that $\sigma_{d}[d] \sigma_{d-1}[d-1] \cdots$ $\sigma_{1}[1] \sigma_{0} \neq 0$. In particular, $\operatorname{Hom}\left(X_{i}, X_{j}[j-i]\right) \neq 0$ and $X_{i} ¥ X_{j}, \forall 0 \leq i<j \leq d$.

Proof From Theorem 4.4 we have that $\sigma_{0} \neq 0$, since the triangle $(*)$ at $i=0$ in Theorem 4.4 is nonsplitting. Suppose that $\sigma_{d}[d] \sigma_{d-1}[d-1] \cdots \sigma_{1}[1] \sigma_{0}=0$; then $\sigma_{d-1}[d-1] \cdots \sigma_{1}[1] \sigma_{0}: X_{0} \rightarrow X_{d}[d]$ factors through $f_{d}[d]: B_{d}[d] \rightarrow$ $X_{d}[d]$, since we have a triangle $X_{d+1}[d] \stackrel{g_{d}[d]}{\longrightarrow} B_{d}[d] \stackrel{f_{d}[d]}{\longrightarrow} X_{d}[d] \stackrel{\sigma_{d}[d]}{\longrightarrow} X_{d+1}[d+1]$. 
Since $\operatorname{Hom}\left(X_{0}, B_{d}[d]\right)=\operatorname{Ext}^{d}\left(X_{0}, B_{d}\right)=0, \sigma_{d-1}[d-1] \cdots \sigma_{1}[1] \sigma_{0}=0$. Similarly, $\sigma_{d-2}[d-2] \cdots \sigma_{1}[1] \sigma_{0}=0$ and, finally, $\sigma_{0}=0$, a contradiction. Now we prove the final statement: we have that $\sigma_{j-1}[j-1] \cdots \sigma_{i} \in \operatorname{Hom}\left(X_{i}, X_{j}[j-i]\right)$ and $\sigma_{j-1}[j-1] \cdots \sigma_{i} \neq 0$. Otherwise $\sigma_{j-1}[j-1] \cdots \sigma_{i}=0$, and hence $\sigma_{d-1}[d-1] \cdots$ $\sigma_{1}[1] \sigma_{0}=0$, a contradiction. Now suppose that $X_{i} \cong X_{j}$ for some $i<j$. Then $\operatorname{Ext}^{k}\left(X_{i}, X_{j}\right)=0$ for $1 \leq k \leq d$, a contradiction. Then $X_{i} ¥ X_{j}$.

Now we state our main result of this section.

Theorem 4.6 Any basic $d$-cluster tilting object in $\mathcal{C}_{d}(\mathcal{H})$ contains exactly $n$ indecomposable direct summands.

To prove the theorem, we need some technical lemmas.

Lemma 4.7 Let $d>1$, and let $X=M[i], Y=N[j]$ be indecomposable objects of degrees $i, j$, respectively, in $\mathcal{C}_{d}(\mathcal{H})$. Suppose that $\operatorname{Hom}(X, Y) \neq 0$. Then one of the following holds:

(1) We have $i=j$ or $j-1$ (provided that $j \geq 1$ ).

(2) We have $i=0, i=d$ (and $M=P$ ) or $d-1$ (provided that $j=0$ ).

Proof Let $d>1$. Firstly we note that, for any indecomposable object $X \in \mathcal{C}_{d}(\mathcal{H})$, $0 \leq \operatorname{deg} X \leq d, \operatorname{deg} X=d$ if and only if $X=P_{i}[d]$ for an indecomposable projective representation $P_{i}$. This implies that $-d \leq \operatorname{deg} Y-\operatorname{deg} X \leq d$ for indecomposable objects $X, Y \in \mathcal{C}_{d}(\mathcal{H})$. Let $X=M[i], Y=N[j]$ be indecomposable objects of degrees $i, j$, respectively, in $\mathcal{C}_{d}(\mathcal{H})$. We have $\operatorname{Hom}(X, Y) \cong$ $\operatorname{Hom}(M, N[j-i])=\bigoplus_{k \in \mathbf{Z}} \operatorname{Hom}_{\mathcal{D}}\left(M, \tau^{-k} N[j-i+k d]\right)=\operatorname{Hom}_{\mathcal{D}}(M, \tau N[j-i-d]) \oplus$ $\operatorname{Hom}_{\mathcal{D}}(M, N[j-i]) \oplus \operatorname{Hom}_{\mathcal{D}}\left(M, \tau^{-1} N[j-i+d]\right)$. The last equality holds due to $-d \leq j-i \leq d$, and $\operatorname{Hom}_{\mathcal{D}}\left(M, \tau^{-k} N[j-i+k d]\right)=0$ for $k \neq-1,0,1$. We divide the calculation of $\operatorname{Hom}(X, Y)$ into three cases:

(1) The case $-d<j-i<d$. We have that $\operatorname{Hom}(X, Y) \cong \operatorname{Hom}_{\mathcal{D}}(M, N[j-i]) \oplus$ $\operatorname{Hom}_{\mathcal{D}}\left(M, \tau^{-1} N[j-i+d]\right)$. The first summand is zero when $j-i \neq 0,1$, while the second is zero when $d+j-i \neq 1$ (equivalently, $d+j-i>1$, since $0<$ $d+j-i<2 d)$.

(2) The case $j-i=-d$. Then $j=0, i=d(M=P)$. Then $\operatorname{Hom}(X, Y)=$ $\operatorname{Hom}_{\mathcal{D}}\left(P, \tau^{-1} N\right)$.

(3) The case $j-i=d$. Then $j=d(N=P), i=0$. Then $\operatorname{Hom}(X, Y)=$ $\operatorname{Hom}_{\mathcal{D}}(M, \tau P) \oplus \operatorname{Hom}_{\mathcal{D}}(M, P[d]) \oplus \operatorname{Hom}_{\mathcal{D}}\left(M, \tau^{-1} P[2 d]\right)=0$.

Therefore, if $\operatorname{Hom}(X, Y) \neq 0$, then $\operatorname{Hom}(M[0], N[j-i]) \neq 0$. Proof of (1). Suppose that $j \geq 1$. Then combining with Case 3 , we have that $-d<j-i<d$. We want to prove that if $j-i \neq 0,1$, then $\operatorname{Hom}(X, Y)=0$, and this will finish the proof of (1). Under the condition $j-i \neq 0,1$, from Case 1 we have that $\operatorname{Hom}(X, Y) \cong \operatorname{Hom}_{\mathcal{D}}\left(M, \tau^{-1} N[j-i+d]\right)$, which is zero for $d+j-i \neq 1$. But if $d+j-i=1$, i.e., $i=d$, then $M=P$ and $j=1$. Then $\operatorname{Hom}_{\mathcal{D}}\left(M, \tau^{-1} N[j-i+d]\right)=$ $\operatorname{Hom}_{\mathcal{D}}\left(P, \tau^{-1} N[1]\right)=0$. We have finished the proof of $(1)$.

Proof of (2) Suppose that $j=0$. Then $-d \leq j-i \leq 0$. From Cases $1-2$ it follows that $i=0, i=d(M=P)$, or $i=d-1$. This finishes the proof of (2). 
Lemma 4.8 If $d>2$, then $\operatorname{Ext}^{2}(M[i], N[i])=0$ for objects $M, N \in \mathcal{H}$ and any $i$.

Proof It is sufficient to prove that $\operatorname{Ext}^{2}(M[0], N[0])=0$. From the definition of the orbit category $\mathcal{D} / \tau^{-1}[d]$ we have that

$$
\operatorname{Ext}^{2}(M[0], N[0])=\operatorname{Hom}(M[0], N[2])=\bigoplus_{k \in \mathbf{Z}} \operatorname{Hom}_{\mathcal{D}}\left(M, \tau^{-k} N[k d+2]\right),
$$

where each summand $\operatorname{Hom}_{\mathcal{D}}\left(M, \tau^{-k} N[k d+2]\right)$ equals 0 , since $k d+2 \geq 2$ or $k d+2 \leq-1$ by the condition $d>2$. Hence, $\operatorname{Ext}^{2}(M[0], N[0])=0$.

Lemma 4.9 Let $d>1$ and $M, N \in \mathcal{H}$. Then $\operatorname{Ext}^{1}(M[0], N[0]) \cong \operatorname{Ext}_{\mathcal{H}}^{1}(M, N)$. Furthermore, any non-split triangle between $M[0]$ and $N[0]$ in $\mathcal{C}_{d}(\mathcal{H})$ is induced from a non-split exact sequence between $M$ and $N$ in $\mathcal{H}$.

Proof Under the condition $d>1$, it is easy to see that $\operatorname{Ext}^{1}(M[0], N[0])=$ $\bigoplus_{k \in \mathbf{Z}} \operatorname{Ext}_{\mathcal{D}}^{1}\left(M, \tau^{-k} N[2 k]\right)=\operatorname{Ext}_{\mathcal{D}}^{1}(M, N)=\operatorname{Ext}_{\mathcal{H}}^{1}(M, N)$. This proves the first statement. Since $\mathcal{H} \subset \mathcal{C}_{d}(\mathcal{H})$ is a (not necessarily full) embedding and any exact short sequence in $\mathcal{H}$ induces a triangle in $\mathcal{C}_{d}(\mathcal{H})$, the final statement then follows from the first statement.

Proof (of Theorem 4.6) We assume that $d>1$, since it was proved in [6] for $d=1$. Let $M=\bigoplus_{i \in I} M_{i}\left[k_{i}\right]$ be a $d$-cluster tilting object in $\mathcal{C}_{d}(\mathcal{H})$, where all $M_{i}$ are indecomposable representations in $\mathcal{H}, 0 \leq k_{i} \leq d$ (when $k_{i}=d, M_{i}$ is projective). One can assume that one of $k_{i}$ is 0 , otherwise one can replace $M$ by a suitable shift of $M$. Denote $v(M)=\max \left\{\left|k_{i}-k_{j}\right| \mid \forall i, j\right\}$. We prove that $|I|=n$ by induction on $v(M)$, where $|I|$ denotes the cardinality of $I$. If $v(M)=0$, i.e., $k_{i}=0$ for all $i$, then $\bigoplus_{i \in I} M_{i}[0]$ is a $d$-cluster tilting object in $\mathcal{C}_{d}(\mathcal{H})$ and hence a tilting object in $\mathcal{H}$. Then $|I|=n$. Now assume that $v(M)=m>0$. Without loss of generality, we assume that $k_{1}=\cdots=k_{t}=m$ and $k_{j}<m$ for $j>t$. From the complement $X_{0}=M_{1}\left[k_{1}\right]$ of $X=M \backslash M_{1}\left[k_{1}\right]$ (here we use $X \backslash X_{1}$ to denote a complement of $X_{1}$ in $X$ for a direct summand $X_{1}$ of $X$ ), by Theorem 4.4 , we have at least $d+1$ complements $X_{j}, j=0, \ldots, d$, which form the triangles $(*)$ in Theorem 4.4. In these triangles, it is easy to see that $f_{i}=0$ if and only if $B_{i}=0$ if and only if $g_{i}=0$. We will prove that there is at least one of complements $X_{j}$ with smaller degree than $m$. At first, we prove this statement for the special case $m=1$. We claim that the degree of $X_{1}$ is 0 or 1 in this case. Otherwise $X_{1}=P[d]$ for some indecomposable projective representation $P$ or $X_{1}=Y[d-1]$ for some indecomposable representation $Y$. Write $X_{0}$ as $Z[1]$, where $Z$ is an indecomposable representation in $\mathcal{H}$. If $X_{1}=P[d]$, then $\operatorname{Hom}\left(X_{1}, X_{0}[d]\right)=\operatorname{Hom}\left(P[d], X_{0}[d]\right) \cong \operatorname{Hom}(P, Z[1])=0$, a contradiction to the fact that $\operatorname{Hom}\left(X_{1}, X_{0}[d]\right) \cong \operatorname{Hom}\left(X_{0}, X_{1}[1]\right)$ is not zero by Theorem 4.4 or Corollary 4.5. If $X_{1}=Y[d-1]$, then $X_{1}$ has degree 1 when $d=2$, and $\operatorname{Hom}\left(X_{1}, X_{0}[d]\right)=\operatorname{Hom}(Y[d-1], Z[d+1]) \cong \operatorname{Ext}^{2}(Y, Z)=0$ by Lemma 4.8 when $d>2$, which also contradicts to the fact that $\operatorname{Hom}\left(X_{1}, X_{0}[d]\right) \cong \operatorname{Hom}\left(X_{0}, X_{1}[1]\right)$ is not zero. This proves the statement that $X_{1}$ has degree 0 or 1 . Now if there are no complements $X_{j}$ of $X$ with degree 0 , then all $X_{j}$ have degree 1 . We prove that any three successive complements, say $X_{0}, X_{1}, X_{2}$, cannot have the same degree. If all 
degrees of $X_{i}, i=0,1,2$, are the same, we can assume that all $X_{i}$ have degree 0 . By Lemma 4.9, we have non-split short exact sequences in $\mathcal{H}$ :

$$
\begin{aligned}
& 0 \longrightarrow X_{1} \longrightarrow B_{0} \longrightarrow X_{0} \longrightarrow 0, \\
& 0 \longrightarrow X_{2} \longrightarrow B_{1} \longrightarrow X_{1} \longrightarrow 0 .
\end{aligned}
$$

From the first short exact sequence we have $\operatorname{Ext}_{\mathcal{H}}^{1}\left(X_{0}, X_{1}\right) ¥ 0$. Applying $\operatorname{Hom}_{\mathcal{H}}\left(X_{0},-\right)$ to the second exact sequence, we have the long exact sequence

$$
\begin{aligned}
\cdots & \rightarrow \operatorname{Ext}_{\mathcal{H}}^{1}\left(X_{0}, X_{2}\right) \rightarrow \operatorname{Ext}_{\mathcal{H}}^{1}\left(X_{0}, B_{1}\right) \rightarrow \operatorname{Ext}_{\mathcal{H}}^{1}\left(X_{0}, X_{1}\right) \\
& \rightarrow \operatorname{Ext}_{\mathcal{H}}^{2}\left(X_{0}, X_{2}\right) \rightarrow \operatorname{Ext}_{\mathcal{H}}^{2}\left(X_{0}, B_{1}\right) .
\end{aligned}
$$

Since $X \oplus X_{0}$ is a $d$-cluster tilting object in $\mathcal{C}_{d}(\mathcal{H})$ and $B_{1} \in \operatorname{add} X$, $\operatorname{Ext}^{1}\left(X_{0}, B_{1}\right)=0$. Hence we have that $\operatorname{Ext}_{\mathcal{H}}^{1}\left(X_{0}, B_{1}\right)=0$ by Lemma 4.9. It follows that $\operatorname{Ext}_{\mathcal{H}}^{1}\left(X_{0}, X_{1}\right)=0$, since $\operatorname{Ext}_{\mathcal{H}}^{2}\left(X_{0}, X_{2}\right)=0$ due to $\mathcal{H}$ being hereditary. It is a contradiction. This finishes the proof for $m=1$.

Now suppose that $m>1$. We will prove that there is at least one of complements $X_{j}$ with smaller degree than $m$. We divide the proof into two cases:

Case 1. All maps $f_{i}$ (equivalently $g_{i}$ ) are nonzero. Now we assume that there are no complements of $X$ with smaller degree than $m$. Then by Lemma 4.7 the degrees of all $X_{i}$ are $m$. If $d>2$, then $\operatorname{Ext}^{2}\left(X_{0}, X_{2}\right)=0$ by Lemma 4.8, a contradiction to Corollary 4.5. If $d=2$, then the same proof as above shows that $\operatorname{Ext}^{1}\left(X_{0}, X_{1}\right)=0$, which contradicts to Corollary 4.5. Therefore, there is a complement of $X$ with smaller degree than $m$.

Case 2. There are some $i$ such that $f_{i}=0$ (equivalently $g_{i}=0$ ). Then $X_{i} \cong$ $X_{i+1}$ [1] for such $i$. It follows that $X_{i+1}$ has smaller degree than $X_{i}$ if $X_{i}$ has a strictly positive degree. Therefore, we have a complement of $X$, say $X_{s}$, such that the degree $k_{1}^{\prime}$ of $X_{s}$ is smaller than $m=k_{1}$. Now we replace $X$ by $X^{\prime}=\left(X \backslash X_{0}\right) \oplus X_{s}$, which is, by Theorem 4.4, a $d$-cluster tilting object in $\mathcal{C}_{d}(\mathcal{H})$ containing $|I|$ indecomposable direct summands. The number of indecomposable direct summands of $X^{\prime}$ with the (maximal) degree $m(=v(M))$ is $t-1$. We repeat the step for the complement $M_{2}\left[k_{2}\right]$ of almost complete tilting object $X^{\prime} \backslash M_{2}\left[k_{2}\right]$, getting a $d$-cluster tilting object $X^{\prime \prime}$ containing $|I|$ indecomposable direct summands, and the number of indecomposable direct summands of $X^{\prime \prime}$ with the (maximal) degree $m(=v(M))$ is $t-2$. Repeating such a step $t$ times, one can get a (basic) $d$-cluster tilting object $T$ containing $|I|$ indecomposable direct summands and $v(T)<v(M)$. By induction, $T$ contains exactly $n$ indecomposable direct summands. Then $|I|=n$.

Remark 4.10 Theorem 4.6 is proved by Thomas [25] for a simply-laced Dynkin quiver $\left(\Gamma, \Omega_{0}\right)$, using the fact that ind $D^{b}(K \vec{\Delta}) \approx \mathbf{Z} \vec{\Delta}$ for a Dynkin quiver $\vec{\Delta}$. This fact does not hold for non-Dynkin quivers. Our proof is more categorical.

Denote by $\mathcal{E}(\mathcal{H})$ the set of isomorphism classes of indecomposable exceptional representations in $\mathcal{H}$. The set $\mathcal{E}\left(\mathcal{C}_{d}(\mathcal{H})\right)$ of isoclasses of indecomposable exceptional objects in $\mathcal{C}_{d}(\mathcal{H})$ is the (disjoint) union of subsets $\mathcal{E}(\mathcal{H})[i], i=0,1, \ldots, d-1$, with $\left\{P_{j}[d] \mid 1 \leq j \leq n\right\}$. A subset $\mathcal{M}$ of $\mathcal{E}\left(\mathcal{C}_{d}(\mathcal{H})\right)$ is called exceptional if, for any 
$X, Y \in \mathcal{M}, \operatorname{Ext}^{i}(X, Y)=0$ for all $i=1, \ldots, d$. Denote by $\mathcal{E}_{+}\left(\mathcal{C}_{d}(\mathcal{H})\right)$ the subset of $\mathcal{E}\left(\mathcal{C}_{d}(\mathcal{H})\right)$ consisting of all indecomposable exceptional objects other than $P_{1}[d], \ldots, P_{n}[d]$.

Now we are ready to define a simplicial complex associated to the $d$-cluster category $\mathcal{C}_{d}(\mathcal{H})$, which is a generalization of the classical cluster complexes of cluster categories $[6,24,26]$.

Definition 4.11 The cluster complex $\Delta^{d}(\mathcal{H})$ of $\mathcal{C}_{d}(\mathcal{H})$ is a simplicial complex which has $\mathcal{E}\left(\mathcal{C}_{d}(\mathcal{H})\right)$ as the set of vertices and has exceptional subsets in $\mathcal{C}_{d}(\mathcal{H})$ as its simplices. The positive part $\Delta_{+}^{d}(\mathcal{H})$ is the subcomplex of $\Delta^{d}(\mathcal{H})$ on the subset $\mathcal{E}_{+}\left(\mathcal{C}_{d}(\mathcal{H})\right)$.

By the definition, the facets (maximal simplices) are exactly the $d$-cluster tilting subsets (i.e., the sets of indecomposable objects of $\mathcal{C}_{d}(\mathcal{H})$ (up to isomorphism) whose direct sum is a $d$-cluster tilting object).

Proposition $4.12(1) \Delta^{d}(\mathcal{H})$ and $\Delta_{+}^{d}(\mathcal{H})$ are pure of dimension $n-1$.

(2) For any sink (or source) $k$, the BGP-reflection functor $\tilde{S_{k}^{+}}$(resp. $\left.\tilde{S_{k}^{-}}\right)$induces an isomorphism between $\Delta^{d}(\mathcal{H})$ and $\Delta^{d}\left(s_{k} \mathcal{H}\right)$. In particular, if $\Gamma$ is a Dynkin diagram and $\Omega$ and $\Omega^{\prime}$ are two orientations of $\Gamma$, then $\Delta^{d}(\mathcal{H})$ and $\Delta^{d}\left(\mathcal{H}^{\prime}\right)$ are isomorphic.

Proof (1) From Theorem 4.6 it follows that any $d$-cluster tilting subset contains exactly $n$ elements. Hence $\Delta^{d}(\mathcal{H})$ is pure of dimension $n-1$. Now suppose that $M=\oplus_{i=1}^{n-1} M_{i}$ is an exceptional object in $\mathcal{C}_{d}(\mathcal{H})$ and that none of the $M_{i}$ are isomorphic to $P_{j}[d]$ for any $j$. In the proof of Theorem 4.6, we proved that not all complements of an almost complete tilting objects have the same degrees. Then $M$ has a complement in $\mathcal{E}_{+}\left(\mathcal{C}_{d}(\mathcal{H})\right)$. This proves that $\Delta_{+}^{d}(\mathcal{H})$ is pure of dimension $n-1$.

(2) Since $\tilde{S_{k}^{+}}$is a triangle equivalence from the $d$-cluster category $\mathcal{C}_{d}(\mathcal{H})$ to $\mathcal{C}_{d}\left(s_{k} \mathcal{H}\right)$, it sends (indecomposable) exceptional objects to (indecomposable) exceptional objects. Thus it induces an isomorphism from $\Delta^{d}(\mathcal{H})$ to $\Delta^{d}\left(s_{k} \mathcal{H}\right)$. The second statement follows from the first statement together with the fact that, for two orientations $\Omega, \Omega^{\prime}$ of a Dynkin graph $\Gamma$, there is a admissible sequence with respect to sinks $i_{1}, \ldots, i_{n}$ such that $\Omega^{\prime}=s_{i_{n}} \cdots s_{i_{1}} \Omega$.

\section{Cluster combinatorics of $\boldsymbol{d}$-cluster categories}

We now define a map $\gamma_{\mathcal{H}}^{d}$ from ind $\mathcal{C}_{d}(\mathcal{H})$ to $\Phi_{\geq-1}^{d}$. Note that any indecomposable object $X$ of degree $i$ in $\mathcal{C}_{d}(\mathcal{H})$ has the form $M[i]$ with $M \in$ ind $\mathcal{H}$, and if $i=d$, then $M=P_{j}$, an indecomposable projective representation.

Definition 5.1 Let $\gamma_{\mathcal{H}}^{d}$ be defined as follows. Let $M[i] \in \operatorname{ind} \mathcal{C}_{d}(\mathcal{H})$, where $M \in$ ind $H$ and $i \in\{1, \ldots, d\}$ (note that if $i=d$, then $M=P_{j}$ for some $j$ ). We set

$$
\gamma_{\mathcal{H}}^{d}(M[i])= \begin{cases}(\underline{\operatorname{dim}} M)^{i+1} & \text { if } M[i] \in \text { ind } \mathcal{H}[i] \text { for some } 0 \leq i \leq d-1 \\ \left(-\alpha_{j}\right)^{1} & \text { if } M[i]=P_{j}[d] .\end{cases}
$$


This map is a kind of extension of correspondence in Gabriel-Kac's Theorem between the indecomposable representations of quivers and positive roots of corresponding Lie- Kac-Moody algebras. It is a bijection if $\Gamma$ is a Dynkin diagram.

We denote by $\Phi_{>0}^{s r}$ the set of real Schur roots of $(\Gamma, \Omega)$, i.e.,

$$
\Phi_{>0}^{s r}=\{\underline{\operatorname{dim}} M M \in \operatorname{ind} \mathcal{E}(\mathcal{H})\} .
$$

Then the map $M \mapsto \underline{\operatorname{dim}} M$ gives a 1-1 correspondence between $\mathcal{E}(\mathcal{H})$ and $\Phi_{>0}^{s r}$ [24].

If we denote by $\Phi_{\geq-1}^{s r, d}$ the set of colored almost positive real Schur roots, which by definition consists of $d$ copies of the set $\Phi_{>0}^{s r}$, together with one copy of the negative simple roots, then the map $\gamma_{\mathcal{H}}^{d}$ gives a bijection from $\mathcal{E}\left(\mathcal{C}_{d}(\mathcal{H})\right)$ to $\Phi_{\geq-1}^{s r, d}$. $\Phi_{\geq-1}^{s r, d}$ contains a subset $\Phi_{>0}^{s r, d}$ consisting of all colored positive real Schur roots. The restriction of $\gamma_{\mathcal{H}}^{d}$ gives a bijection from $\mathcal{E}_{+}\left(\mathcal{C}_{d}(\mathcal{H})\right)$ to $\Phi_{>0}^{s r, d}$.

Since $\mathcal{E}(\mathcal{H}) \longrightarrow \Phi_{>0}^{s r}: M \mapsto \underline{\operatorname{dim}} M$ is a bijection, we use $M_{\beta}$ to denote the unique indecomposable exceptional representation in $\mathcal{H}$ whose dimension vector is $\beta$. From Proposition 4.2 it follows that $\left.\gamma_{\mathcal{H}}^{d}\left(M_{\beta}[i]\right)\right)=\beta^{i+1}$ for any $0 \leq i \leq d-1$. We sometimes use $M_{\beta^{i+1}}$ to denote the unique preimage of a colored almost positive real Schur root $\beta^{i+1}$ under $\gamma_{\mathcal{H}}^{d}$.

We now prepare to define a simplicial complex $\Delta^{d, \mathcal{H}}(\Phi)$ associated with any root system $\Phi$, which turns out to be isomorphic to the cluster complex $\Delta^{d}(\mathcal{H})$ of the $d$-cluster category $\mathcal{C}_{d}(\mathcal{H})$. When $\Gamma$ is a Dynkin graph, taking an alternating orientation $\Omega_{0}$ of $\Gamma$, this complex $\Delta^{d, \mathcal{H}_{0}}(\Phi)$ is the generalized cluster complex $\Delta^{d}(\Phi)$ defined by Fomin and Reading [12].

First of all, we define the " $d$-compatibility degree" on any pair of colored almost positive real Schur roots.

Definition 5.2 For any pair of colored almost positive real Schur roots $\alpha, \beta$, the $d$-compatibility degree of $\alpha, \beta$ is defined as follows:

$$
(\alpha \| \beta)_{d, \mathcal{H}}=\operatorname{dim}_{\operatorname{End} M_{\alpha}}\left(\operatorname{Ext}^{1}\left(M_{\alpha}, \bigoplus_{i=0}^{i=d-1} M_{\beta}[i]\right)\right),
$$

where $\quad \operatorname{dim}_{\operatorname{End} M_{\alpha}}\left(\operatorname{Ext}^{1}\left(M_{\alpha}, \bigoplus_{i=0}^{i=d-1} M_{\beta}[i]\right)\right)$ denotes the length of $\operatorname{Ext}^{1}\left(M_{\alpha}, \bigoplus_{i=0}^{i=d-1} M_{\beta}[i]\right)$ as a right End $M_{\alpha}$-module. When $d>1$, End $M_{\alpha}$ is a division algebra by Proposition 4.2(3), and this length equals the dimension of $\operatorname{Ext}^{1}\left(M_{\alpha}, \bigoplus_{i=0}^{i=d-1} M_{\beta}[i]\right)$ over the division algebra End $M_{\alpha}$.

Remark 5.3 When $\Gamma$ is a Dynkin diagram with trivial valuation and $\Omega_{0}$ is an alternating orientation of $\Gamma$, this compatibility degree is defined in [25]. When $d=1$ and $\Gamma$ is a Dynkin diagram, we recover the classical compatibility degree defined in $[6,27]$.

Theorem 5.4 (1) For any pair of colored almost positive real Schur roots $\alpha, \beta$, we have:

(a) $(\alpha \| \beta)_{d, \mathcal{H}}=\left(\sigma_{k, d}(\alpha) \| \sigma_{k, d}(\beta)\right)_{d, s_{k}} \mathcal{H}$ if $k$ is a sink (or a source). 
(b) $(\alpha \| \beta)_{d, \mathcal{H}}=\left(R_{d, \Omega}(\alpha) \| R_{d, \Omega}(\beta)\right)_{d, \mathcal{H}}$.

(c) $(\alpha \| \beta)_{d, \mathcal{H}}=0$ if and only if $(\beta \| \alpha)_{d, \mathcal{H}}=0$.

(2) For any almost positive real Schur root $\beta,\left(\left(-\alpha_{i}\right)^{1} \|(\beta)^{l}\right)_{d, \mathcal{H}}=0$ if and only if $\max \left\{n_{i}(\beta), 0\right\}=0$, where $n_{i}(\beta)$ is the coefficient of $\alpha_{i}$ in the expansion of $\beta$ in terms of the simple roots $\alpha_{1}, \ldots, \alpha_{n}$.

Proof (1) Let $\alpha, \beta$ be two colored almost positive real Schur roots.

(a) We prove it for the case $k$ is a sink, the proof for source is similar. It is easy to check that the following diagram is commutative:

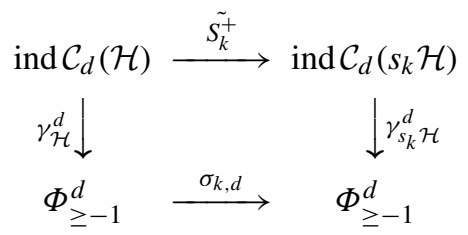

Hence we have that

$$
\begin{aligned}
\left(\sigma_{k, d}(\alpha) \| \sigma_{k, d}(\beta)\right)_{d, s_{k} \mathcal{H}} & =\operatorname{dim}_{\operatorname{End}_{S_{k}^{+}}\left(M_{\alpha}\right)} \operatorname{Ext}^{1}\left(\tilde{S_{k}^{+}}\left(M_{\alpha}\right), \bigoplus_{i=0}^{i=d-1} \tilde{S_{k}^{+}}\left(M_{\beta}\right)[i]\right) \\
& =\operatorname{dim}_{\operatorname{End} M_{\alpha}} \operatorname{Ext}^{1}\left(M_{\alpha}, \bigoplus_{i=0}^{i=d-1} M_{\beta}[i]\right)=(\alpha \| \beta)_{d, \mathcal{H}}
\end{aligned}
$$

(b) As we mentioned before, the shift functor [1] of $\mathcal{C}_{d}(\mathcal{H})$ is an auto-equivalence. We now check that the following diagram commutes:

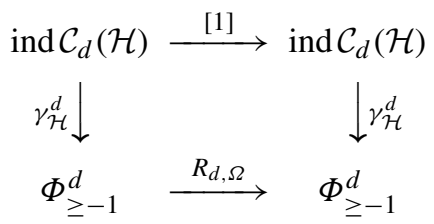

By Proposition 2.2, any indecomposable object in $\mathcal{C}_{d}(\mathcal{H})$ is of the form $X[i]$ with $X$ an indecomposable representation in $\mathcal{H}$ and with $0 \leq i \leq d-1$ or of the form $P_{j}[d]$. Denote $\underline{\operatorname{dim}} X=\alpha$. If $i \leq d-2$, then $R_{d, \Omega} \gamma_{\mathcal{H}}^{d}(X[i])=R_{d, \Omega}\left((\alpha)^{i+1}\right)=$ $(\alpha)^{i+2}=\gamma_{\mathcal{H}}^{d}[1](X[i])$. We will prove the equality for other indecomposable objects in $\mathcal{C}_{d}(\mathcal{H})$. Firstly, we have that $\left.R_{d, \Omega} \gamma_{\mathcal{H}}^{d}\left(P_{j}[d-1]\right)=R_{d, \Omega}\left(\underline{\operatorname{dim}} P_{j}\right)^{d}\right)=\left(-\alpha_{j}\right)^{1}$ and $\gamma_{\mathcal{H}}^{d}[1]\left(P_{j}[d-1]\right)=\left(-\alpha_{j}\right)^{1}$. Hence $R_{d, \Omega} \gamma_{\mathcal{H}}^{d}\left(P_{j}[d-1]\right)=\gamma_{\mathcal{H}}^{d}[1]\left(P_{j}[d-1]\right)$. Secondly, for any $X[d-1]$ with $X$ not being projective, we have $\tau X \in$ ind $\mathcal{H}$. We have that $R_{d, \Omega} \gamma_{\mathcal{H}}^{d}(X[d-1])=R_{d, \Omega}\left((\alpha)^{d}\right)=\left(R_{\Omega}(\alpha)\right)^{1}$ and $\gamma_{\mathcal{H}}^{d}[1](X[d-1])=$ $\gamma_{\mathcal{H}}^{d}(X[d])=\gamma_{\mathcal{H}}^{d}\left(\tau^{-1}[d] \tau X\right)=\gamma_{\mathcal{H}}^{d}(\tau X)=\left(R_{\Omega}(\alpha)\right)^{1}$. The last equality holds, since $\underline{\operatorname{dim}} \tau X=R_{\Omega}(\underline{\operatorname{dim}} X)$ (cf. Sect. 3.1). This proves that $R_{d, \Omega} \gamma_{\mathcal{H}}^{d}(X[d-1])=$ $\gamma_{\mathcal{H}}^{d}[1](X[d-1])$. For $P_{j}[d]$, the proof is similar. We finish the proof of the commutativity of the diagram. 
It follows that

$$
\begin{aligned}
\left(R_{d, \Omega}(\alpha) \| R_{d, \Omega}(\beta)\right)_{d, \mathcal{H}} & =\operatorname{dim}_{\operatorname{End} M_{\alpha}[1]} \operatorname{Ext}^{1}\left(M_{\alpha}[1], \bigoplus_{i=0}^{i=d-1} M_{\beta}[1][i]\right) \\
& =\operatorname{dim}_{\operatorname{End} M_{\alpha}} \operatorname{Ext}^{1}\left(M_{\alpha}, \bigoplus_{i=0}^{i=d-1} M_{\beta}[i]\right)=(\alpha \| \beta)_{d, \mathcal{H}},
\end{aligned}
$$

where the second equality follows from the fact that [1] is an equivalence.

(c) Let $X, Y \in \mathcal{C}_{d}(\mathcal{H})$ with $\operatorname{Ext}^{i}(X, Y)=0$ for any $1 \leq i \leq d$. Then by the Calabi-Yau property of $\mathcal{C}_{d}(\mathcal{H})$ we have that, for any $1 \leq j \leq d, \operatorname{Ext}^{j}(Y, X) \cong$ $\operatorname{Ext}^{d-j+1}(X, Y)=0$. This proves (c).

(2) We first prove the necessity: Let $\beta$ be an almost positive real Schur root with $\left(\left(-\alpha_{i}\right)^{1} \|(\beta)^{l}\right)_{d, \mathcal{H}}=0$. If $\beta$ is a negative simple root and $l=1$, we easily have that $\max \left\{n_{i}(\beta), 0\right\}=0$. Now we assume that $\beta$ is a positive real Schur root. From the condition $\left(\left(-\alpha_{i}\right)^{1} \|(\beta)^{l}\right)_{d, \mathcal{H}}=0$ we have $\operatorname{Ext}^{j}\left(P_{i}[d], M_{\beta^{l}}\right)=0$, i.e., $\operatorname{Ext}^{j}\left(P_{i}[d], M_{\beta}[l-1]\right)=0$ for any $1 \leq j \leq d$. Since $1 \leq l \leq d$, we have $1 \leq j \leq d$, where $j=d+1-l$. Now we have that $0=$ $\operatorname{Ext}^{j}\left(P_{i}[d], M_{\beta}[l-1]\right) \cong \operatorname{Hom}\left(P_{i}[d], M_{\beta}[l+j-1]\right) \cong \operatorname{Hom}\left(P_{i}, M_{\beta}\right)$. Hence $n_{i}(\beta)=\operatorname{dim}_{\text {End } P_{i}} \operatorname{Hom}\left(P_{i}, M_{\beta}\right)=0$.

Now we prove the other direction. Suppose that $\beta$ is an almost positive real Schur root with $\max \left\{0, n_{i}(\beta)\right\}=0$. Firstly, if $\beta$ is the negative of a simple root, say $\left(-\alpha_{j}\right)^{1}$, then

$$
\begin{aligned}
\left(\left(-\alpha_{i}\right)^{1} \|\left(-\alpha_{j}\right)^{1}\right)_{d, \mathcal{H}} & =\operatorname{dim}_{\operatorname{End}\left(P_{i}[d]\right)} \operatorname{Ext}^{1}\left(P_{i}[d], \bigoplus_{k=0}^{k=d-1} P_{j}[d][k]\right) \\
& =\operatorname{dim}_{\operatorname{End}\left(P_{i}[d]\right)} \operatorname{Ext}^{1}\left(P_{i}, \bigoplus_{k=0}^{k=d-1} P_{j}[k]\right) \\
& =\operatorname{dim}_{\operatorname{End}\left(P_{i}[d]\right)} \operatorname{Ext}^{1}\left(P_{i}, P_{j}[d-1]\right),
\end{aligned}
$$

the last equality following from Proposition 4.2(4). But $\operatorname{Ext}^{1}\left(P_{i}, P_{j}[d-1]\right) \cong$ $\operatorname{Hom}\left(P_{i}, P_{j}[-1][d+1]\right) \cong D \operatorname{Hom}\left(P_{j}[-1], P_{i}\right) \cong D \operatorname{Ext}^{1}\left(P_{j}, P_{i}\right)=0$. This proves that $\left(\left(-\alpha_{i}\right)^{1} \|\left(-\alpha_{j}\right)^{1}\right)_{d, \mathcal{H}}=0$. Now we assume that $\beta$ is a positive real Schur root and $l$ is a positive integer not exceeding $d$. We will prove that $\left(\left(-\alpha_{i}\right)^{1} \|(\beta)^{l}\right)_{d, \mathcal{H}}=0$ under the condition that $n_{i}(\beta)=0$. We can assume that $d>1$, since, for $d=1$, the corresponding result is proved in [26]. From the condition $n_{i}(\beta)=0$ it follows that $\operatorname{Hom}_{\mathcal{H}}\left(P_{i}, M_{\beta}\right)=0$ and then $\operatorname{Hom}\left(P_{i}, M_{\beta}\right)=0$. Hence $\operatorname{Ext}^{1}\left(P_{i}[d], M_{\beta}[d-\right.$ $1]) \cong \operatorname{Hom}\left(P_{i}, M_{\beta}\right)=0$. We will prove that $\operatorname{Ext}^{j}\left(P_{i}[d], M_{\beta^{l}}\right)=0$ for $1 \leq j \leq d$. Now given such $j, \operatorname{Ext}^{j}\left(P_{i}[d], M_{\beta^{l}}\right)=\operatorname{Ext}^{j}\left(P_{i}[d], M_{\beta}[l-1]\right)=\operatorname{Ext}^{1}\left(P_{i}[d]\right.$, $\left.M_{\beta}[l+j-2]\right) \cong \operatorname{Ext}^{1}\left(P_{i}, M_{\beta}[l+j-d-2]\right)$. Since $1 \leq l \leq d, 1 \leq j \leq d$, we have $-d \leq l+j-d-2 \leq d-2$. Then we have that $\operatorname{Ext}^{j}\left(P_{i}[d], M_{\beta^{l}}\right)=0$, which follows from Proposition 4.2(4) for $l+j-d-2 \neq-1$ and from the fact that $\operatorname{Ext}^{1}\left(P_{i}, M_{\beta}[-1]\right) \cong \operatorname{Hom}\left(P_{i}, M_{\beta}\right)=0$ for $l+j-d-2=-1$. 
Definition 5.5 Let $\Phi$ be the root system corresponding to $\Gamma$ and $\mathcal{H}$ the category of representations of the valued quiver $(\Gamma, \Omega)$.

(1) Any pair $\alpha, \beta$ of almost positive real Schur roots is called $d$-compatible if ( $\alpha \|$ $\beta)_{d, \mathcal{H}}=0$; a subset of $\Phi_{\geq-1}^{s r, d}$ is called $d$-compatible if any two elements of this subset are compatible.

(2) The simplicial complex $\Delta^{d, \mathcal{H}}(\Phi)$ associated to $\Phi$ and $\mathcal{H}$ is a complex which has $\Phi_{\geq-1}^{s r, d}$ as the set of vertices. Its simplices are $d$-compatible subsets of $\Phi_{\geq-1}^{s r, d}$. The subcomplex of $\Delta^{d, \mathcal{H}}(\Phi)$ which has $\Phi_{>0}^{s r, d}$ as the set of vertices is denoted by $\Delta_{+}^{d, \mathcal{H}}(\Phi)$. We call $\Delta^{d, \mathcal{H}}(\Phi)$ the generalized cluster complex associated to $\Phi$ and $\mathcal{H}$.

Remark 5.6 Given a graph $\Gamma$, we have the corresponding root system $\Phi$. Since the set of real Schur roots of $\Phi$ depends on the category ind $\mathcal{H}$, equivalently, on the orientation $\Omega$ of $\Gamma$, the generalized cluster complexes $\Delta^{d, \mathcal{H}}(\Phi)$ are possibly nonisomorphic for different orientations of $\Gamma$, but they are isomorphic to each other if $\Gamma$ is a Dynkin diagram by Proposition 4.12(2) and the following theorem.

Theorem 5.7 (1) Let $\Gamma$ be a valued graph and $\Phi$ the corresponding root system. Let $\Omega$ be an admissible orientation of $\Gamma$. Then $\gamma_{\mathcal{H}}^{d}$ provides an isomorphism from the simplicial complex $\Delta^{d}(\mathcal{H})$ to the generalized cluster complex $\Delta^{d, \mathcal{H}}(\Phi)$, which sends vertices to vertices and $k$-faces to $k$-faces.

(2) The restriction of $\gamma_{\mathcal{H}}^{d}$ to $\Delta_{+}^{d}(\mathcal{H})$ gives an isomorphism from $\Delta_{+}^{d}(\mathcal{H})$ to $\Delta_{+}^{d, \mathcal{H}}(\Phi)$.

(3) If $\Gamma$ is a Dynkin graph, and $\Omega_{0}$ is an alternating orientation of $\Gamma$, then $\Delta^{d, \mathcal{H}_{0}}(\Phi)$ is the generalized cluster complex $\Delta^{d}(\Phi)$ defined by Fomin and Reading in [12].

Proof (1) $\gamma_{\mathcal{H}}^{d}$ provides a bijection from the vertices of $\Delta^{d}(\mathcal{H})$ to that of $\Delta^{d, \mathcal{H}}(\Phi)$. For any pair of colored almost positive real Schur roots $\alpha^{k}, \beta^{l}$, they are $d$-compatible if and only if $M_{\alpha^{k}} \oplus M_{\beta^{l}}$ is an exceptional object, where $M_{\alpha^{k}}$ and $M_{\beta^{l}}$ are the exceptional objects corresponding to $\alpha^{k}, \beta^{l}$ respectively under the map $\gamma_{\mathcal{H}}^{d}$. Hence $\gamma_{\mathcal{H}}^{d}$ is an isomorphism from $\Delta^{d}(\mathcal{H})$ to $\Delta^{d, \mathcal{H}}(\Phi)$.

(2) This is a direct consequence of (1).

(3) This is a direct consequence of Theorems 3.9 and 5.4.

From Theorem 5.7 one can translate results from each side. For example, one gets the number of $d$-cluster tilting objects in $\mathcal{C}_{d}(\mathcal{H})$ from the number of facets of generalized cluster complexes of finite root systems [12].

Corollary 5.8 (1) The generalized cluster complex $\Delta^{d, \mathcal{H}}(\Phi)$ and its subcomplex $\Delta_{+}^{d, \mathcal{H}}(\Phi)$ are pure of dimension $n-1$.

(2) Let $(\Gamma, \Omega)$ be a connected Dynkin quiver and $\Phi$ the root system corresponding to $\Gamma$. Then the number of $d$-cluster tilting objects of $\mathcal{C}_{d}(\mathcal{H})$ is $\prod_{i} \frac{d h+e_{i}+1}{e_{i}+1}$, where $h$ is the Coxeter number of $\Phi$, and $e_{1}, \ldots, e_{n}$ are the exponents of $\Phi$. 
(3) Let $(\Gamma, \Omega)$ be a connected Dynkin quiver and $\Phi$ the corresponding root system. Then the number of complements of any almost complete tilting object in $\mathcal{C}_{d}(\mathcal{H})$ is $d+1$.

Proof (1) It follows from Proposition 4.12(1) and Theorem 5.7(1).

(2) From Theorem 5.7(1) and Proposition 8.4 in [12] it follows that the statement holds for the $d$-cluster category $\mathcal{C}_{d}\left(\mathcal{H}_{0}\right)$ of $\Omega_{0}$. Then by Proposition 4.12(2) the statement holds for a $d$-cluster category $\mathcal{C}_{d}(\mathcal{H})$ corresponding to an arbitrary orientation $\Omega$.

(3) From Theorem 5.7(1) and Proposition 3.10 in [12] it follows that the number of complements of any almost complete tilting object in $\mathcal{C}_{d}\left(\mathcal{H}_{0}\right)$ is $d+1$. Hence by Proposition 4.12(2) the number of complements of any almost complete tilting object in $\mathcal{C}_{d}(\mathcal{H})$ is $d+1$.

Remark 5.9 Corollary 5.8(1) generalizes Theorem 2.9 in [12] to infinite root systems.

Remark 5.10 (1) From Corollary 5.8(2) for $d=1$, combining with the result in [6] (see also [21]), in which the cluster tilting subcategories in $\mathcal{D}$ are proved to be in one-to-one correspondence with the cluster tilting modules in cluster categories by the projection $\pi$, we have an explanation on why the number of cluster tilting subcategories (i.e., Ext-configurations in [16]) in $\mathcal{D}$ is the same as the number of facets of $\Delta(\Phi)$.

(2) Corollary 5.8(3) is proved by Thomas [25] for an alternating simply-laced Dynkin quiver $\left(\Gamma, \Omega_{0}\right)$, using a different approach.

Acknowledgements The author would like to thank Claus Michael Ringel and Osamu Iyama for their helpful suggestions on cluster complexes and the formalism of the paper and to thank Bernhard Keller for informing the author of Y. Palu's work. After completing this work, I was told by Bernhard Keller and Robert Marsh that H. Thomas had some similar results for simply-laced Dynkin type [25]. I thank them very much for this!

The author would like to thank the referees for their useful suggestions to improve the paper.

\section{References}

1. Assem, I., Brstle, T., Schiffler, R., \& Todorov, G. (2006). $m$-cluster categories and $m$-replicated algebras. Preprint arXiv:math.RT/0608727.

2. Athanasiadis, C., \& Tzanaki, E. (2006). Shellability and higher Cohen-Macaulay connectivity of generalized cluster complexes. Preprint arXiv:math.CO/0606018.

3. Baur, K., \& Marsh, R. A geometric description of $m$-cluster categories. Transactions of the AMS, to appear. Preprint arXiv:math.RT/0610512.

4. Buan, A., \& Marsh, R. (2006) . Cluster-tilting theory. In J. de la Peña \& R. Bautista (Eds.), Trends in representation theory of algebras and related topics, contemporary mathematics (Vol. 406, p. 1-30).

5. Buan, A., Marsh, R., \& Reiten, I. (2004). Cluster mutation via quiver representations. Comment. Math. Helv., to appear. Preprint arXiv:math.RT/0412077.

6. Buan, A., Marsh, R., Reineke, M., Reiten, I., \& Todorov, G. (2006). Tilting theory and cluster combinatorics. Adv. Math., 204, 572-618.

7. Caldero, P., \& Chapoton, F. (2006). Cluster algebras as Hall algebras of quiver representations. Comment. Math. Helv., 81, 595-616.

8. Caldero, P., \& Keller, B. From triangulated categories to cluster algebras. Invent. Math., to appear. Preprint arXiv:math.RT/0506018. 
9. Caldero, P., Chapoton, F., \& Schiffler, R. (2006). Quivers with relations arising from clusters $\left(A_{n}\right.$ case). Trans. Am. Math. Soc., 358, 1347-1364.

10. Dlab, V., \& Ringel, C. M. Indecomposable representations of graphs and algebras. Mem. Am. Math. Soc. 591 (1976).

11. Fomin, S., \& Reading, N. (2004). Root system and generalized associahedra. In Lecture notes for the IAS/Park City graduate summer school in geometric combinatorics.

12. Fomin, S., \& Reading, N. (2005). Generalized cluster complexes and Coxeter combinatorics. IMRN, 44, 2709-2757.

13. Fomin, S., \& Zelevinsky, A. (2002). Cluster algebras I: foundations. J. Am. Math. Soc., 15(2), 497529.

14. Fomin, S., \& Zelevinsky, A. (2003). Y-system and generalized associahedra. Ann. Math., 158, 9771018.

15. Fomin, S., \& Zelevinsky, A. (2003). Cluster algebras II: finite type classification. Invent. Math., 154(1), 63-121.

16. Iyama, O. (2007). Higher dimensional Auslander-Reiten theory on maximal orthogonal subcategories. Adv. Math., 210(1), 22-50.

17. Iyama, O. (2005). Maximal orthogonal subcategories of triangulated categories satisfying Serre duality. Mathematisches Forschungsinstitut Oberwolfach Report, no. 6 (pp. 353-355).

18. Iyama, O., \& Yoshino, Y. Mutations in triangulated categories and rigid Cohen-Macaulay modules. Preprint arXiv:math.RT/0607736.

19. Keller, B. (2005). Triangulated orbit categories. Documenta Math., 10, 551-581.

20. Keller, B., \& Reiten, I. (2007). Cluster-tilted algebras are Gorenstein and stably Calabi-Yau. Adv. Math. 211(1), 123-151.

21. Koenig, S., \& Zhu, B. From triangulated categories to Abelian categories-cluster tilting in a general framework. Math. Z., to appear. See also preprint arXiv:math.RT/0605100.

22. Marsh, R., Reineke, M., \& Zelevinsky, A. (2003). Generalized associahedra via quiver representations. Trans. Am. Math. Soc., 355(10), 4171-4186.

23. Palu, Y. Ph.D. Thesis, in preparation.

24. Ringel, C. M. (2007). Some remarks concerning tilting modules and tilted algebras. Origin. Relevance. Future. An appendix to the Handbook of tilting theory, L. Angeleri-Hügel, D. Happel \& H. Krause (Eds.). LMS Lecture Notes Series (Vol. 332). Cambridge University Press.

25. Thomas, H. (2005). Defining an m-cluster category. Preprint.

26. Zhu, B. (2007). BGP-reflection functors and cluster combinatorics. J. Pure Appl. Algebra, 209, 497506.

27. Zhu, B. (2006). Equivalences between cluster categories. J. Algebra, 304, 832-850. 